\title{
Parkinson's Disease-Associated Mutant LRRK2-Mediated Inhibition of miRNA Activity is Antagonized by TRIM32
}

\author{
Laura Gonzalez-Cano $^{1}$ • Ingeborg Menzl $^{1} \cdot$ Johan Tisserand $^{1} \cdot$ Sarah Nicklas $^{1}$ • \\ Jens C. Schwamborn ${ }^{1}$
}

Received: 20 December 2016 / Accepted: 21 April 2017 /Published online: 15 May 2017

(C) The Author(s) 2017. This article is an open access publication

\begin{abstract}
Parkinson's disease (PD) is the second most common neurodegenerative disorder. Accumulating evidences suggest that PD might have a strong neurodevelopmental component. Among the genetic cases, mutations in the leucine-rich repeat kinase 2 (LRRK2) are well known to be disease causing. Although the molecular mechanism of the pathogenic LRRK2 function is not fully clear, inhibition of microRNA (miRNA) activity has been suggested to be among the pathogenic LRRK2 targets. Here, we demonstrate that the miRNA activity inhibition function of pathogenic LRRK2 is directly antagonized by the neuronal cell fate determinant TRIM32. These findings suggest that TRIM32 might be a modifier for PD and could be a novel therapeutic target.
\end{abstract}

Keywords Parkinson's disease · TRIM32 - LRRK2 - miRNA activity $\cdot$ Neuronal differentiation

\section{Introduction}

Parkinson's disease (PD) is the second most common neurodegenerative disorder [1]. The main pathologic hallmark of PD is the degeneration of dopaminergic neurons in the substantia nigra of the midbrain, but it is now accepted that the disease is way more complex and that multiple brain regions and organs beyond the brain are affected [2-4].

Electronic supplementary material The online version of this article (doi:10.1007/s12035-017-0570-y) contains supplementary material, which is available to authorized users.

Jens C. Schwamborn

jens.schwamborn@uni.lu

1 Luxembourg Centre for Systems Biomedicine (LCSB), University of Luxembourg, 6, Avenue du Swing, 4367 Belvaux, Luxembourg
Accumulating evidences even suggest that PD might have a strong neurodevelopmental component, implying that deregulated processes during embryonic development lead to PD, which determines the anlagen or susceptibility to develop the disease [5-7]. This anlage might be compensated for a long time before symptoms develop at higher ages.

Currently, about $15 \%$ of PD patients have a monogenic disease caused by one of the known mutations in the 15 associated genes; additionally, 25 genetic risk factors have been identified [8]. Leucine-rich repeat kinase 2 (LRRK2) is probably the best studied PD-associated gene [9]. Interestingly, PD-associated mutations in this gene only cause the disease in about $30 \%$ of the carriers [10]. The latter suggests that other genetic or environmental factors strongly contribute to the development of PD. Recently, it has been demonstrated in Drosophila that pathogenic LRRK2 inhibits microRNA (miRNA)-mediated translational repression [11]. Interestingly, important cell cycle regulators were affected by this altered translational control. This observation further supports the concept that PD-associated genes play a significant function in developmentally important processes like cell cycle control. It is tempting to speculate that pathogenic LRRK2, via miRNA activity modification, affects the cell cycle in neural stem cells and thereby alters the fate specification of neurons during development. This effect of pathogenic LRRK 2 might result only in a slight delay in the neuronal differentiation process. However, eventually this delay is probably enough to alter the complex neuronal system, which might be the basis for increasing neurodegeneration susceptibility at a later stage.

In contrast to LRRK2, the neuronal cell fate determinant TRIM32 has been described as an activator of miRNAmediated translational repression [12, 13]. TRIM32 belongs to the TRIM-NHL family of proteins that is characterized by the presence of an N-terminal RING finger, one or two B boxes, a coiled-coil region, and a C-terminal NHL domain 
[14]. This conserved protein family has been implicated in diverse biological processes, such as developmental timing, cell cycle progression, transcriptional regulation, and apoptosis [15]. Previously, we have shown that TRIM32 suppresses proliferation and induces neuronal differentiation in NSCs from embryonic [13, 16, 17] and adult mouse brain [18]. Through its C-terminal NHL domain, TRIM32 directly binds to miRNA-associated proteins of the Argonaute family, which leads to enhanced activity of specific microRNAs including Let-7a [12, 13]. Interestingly, TRIM32 has been implicated as regulator or target of the PD-associated genes alpha-synuclein and parkin $[19,20]$.

Here, we demonstrate that mammalian LRRK2 directly interacts with the Argonaute- 2 protein and that pathogenic mutant LRRK2 inhibits the activity of the miRNA Let-7a. We further show that the effect of pathogenic LRRK2 is directly antagonized by TRIM32. These results suggest TRIM32 as a novel target for Parkinson's disease-modifying therapies.

\section{Results and Discussion}

\section{LRRK2, TRIM32, and Ago2 Interact In Vitro and In Vivo}

Previously, it has been shown in Drosophila that LRRK2 interacts with Argonaute proteins [11]. In the first set of experiments, we wanted to confirm if this interaction is conserved in mammals. If so, we aimed at addressing whether it depends on the presence of the PD-associated G2019S mutation in LRRK2, which increases the kinase activity [21], or the kinase dead mutation, D2016A. Furthermore, we were interested in determining the presence of TRIM32 in the LRRK2/Ago2 complex. Therefore, we co-transfected HEK293T cells, which endogenously express Ago2, with plasmids encoding for GFP-TRIM32 together with different Flag-LRRK2 constructs (Fig. 1a, Supplementary Fig. 1A). Via immunoprecipitation (IP) assays, we saw that LRRK2 is able to co-precipitate TRIM32 and that Ago2 co-precipitates with LRRK2 (Fig. 1a, Supplementary Fig. 1A). None of these interactions was significantly affected by the presence of LRRK2 mutations. Furthermore, the co-expression of TRIM32 also had no detectable effect on the stability of LRRK2 or Ago2 (Supplementary Fig. 1b).

After demonstrating the existence of a LRRK2/Ago2/ TRIM32 complex in vitro, we wanted to verify this interaction with endogenously present proteins in vivo. Therefore, we used brain lysates from mice of 2, 5, and 7 months of age. Interestingly, we observed a strong downregulation of LRRK2 during this time period (Fig. 1b). A similar ageassociated downregulation of LRRK2 has been observed before in the mouse spleen [22]. This observation further supports the hypothesis that LRRK2 has an important role during development. Again, via IP assays with antibodies directed against either LRRK2 or Ago2, we were able to show an interaction between LRRK2, Ago2, and TRIM32 (Fig. 1b). None of these interactions was significantly affected by the age of the animals. With this first set of experiments, we have provided evidences that LRRK2, Ago2, and TRIM32 form a complex in vitro and in vivo.

\section{TRIM32 and Mutant LRRK2 Bind Independently at the RNA-Induced Silencing Complex}

In the next step, we wanted to assess whether the interaction of TRIM32 and LRRK2 with the Ago2-containing RISC is mutually dependent and whether the pathogenic LRRK2G2019S mutation has any influence on this interaction. Therefore, we generated double-transgenic mice that either express the wild-type version of TRIM32 or present a TRIM32 knockout [23]. Additionally, these mice express either wild-type LRRK2 (non-carrier, nc) or LRRK2-G2019S [24]. We used brain lysates from these mice to conduct an IP with anti-Ago2 antibodies. However, regardless of the presence or absence of TRIM32, the Ago2-LRRK2 interaction was robustly present (Fig. 2a). Moreover, the interaction between Ago 2 and TRIM32 was not influenced by the presence or absence of the LRRK2-G2019S mutation (Fig. 2a).

Both LRRK2 and TRIM32 can bind to Ago2 independently of each other but have reported opposite effects on the activity of the RISC. Due to that, we were aiming at elucidating whether they probably compete for binding to Ago2. In order to address this question, we expressed TRIM32 and LRRK2G2019S in the ratio 1:1, 1:10, or 10:1 in HEK293T cells (Fig. 2b). Interestingly, we observed the highest levels of TRIM32 when it is co-expressed with a ten times excess of LRRK2 (Fig. 2b, c). We speculate that high LRRK2 levels might inhibit the degradation of TRIM32 via autoubiquitination and thereby increase its stability. As expected, highest LRRK2 expression levels were observed when it is expressed at a ten times excess (Fig. 2b, d). However, this is significantly reduced when it is co-expressed with TRIM32 (10× LRRK2, $1 \times$ TRIM32). This observation might indicate that TRIM32 downregulates LRRK2 when it is overexpressed, which probably is mediated via an ubiquitin ligase activity of TRIM32 towards LRRK2. However, we were not able to detect an effect of TRIM32 on the LRRK2 levels in the previous experiments (Fig. 1, Supplementary Fig. 1), indicating that this effect probably strongly depends on the levels in which both proteins are present. Strikingly, when immunoprecipitating LRRK2 or Ago2 from these cell lysates, we found that the interactions between Ago2 and TRIM32 as well as between Ago2 and LRRK2 are independent of their expression levels (Fig. 2e). Specificity of the immunoprecipitation assays was verified by control immunoprecipitations with $\mathrm{IgG}$ isotypenegative control antibodies (Supplementary Fig. 2B). Based 


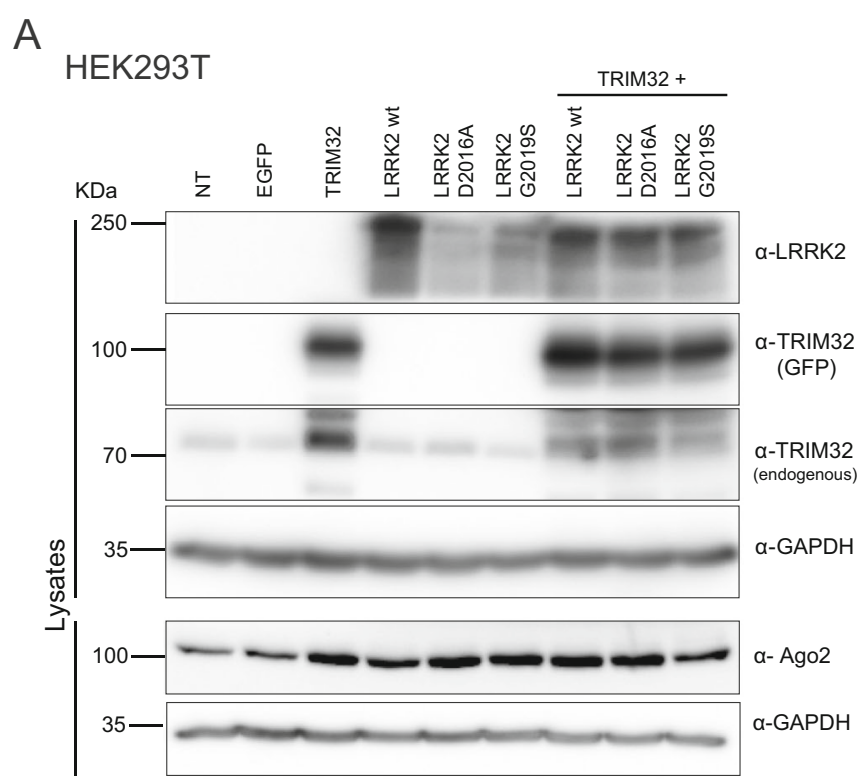

B

\section{Brain lysates}

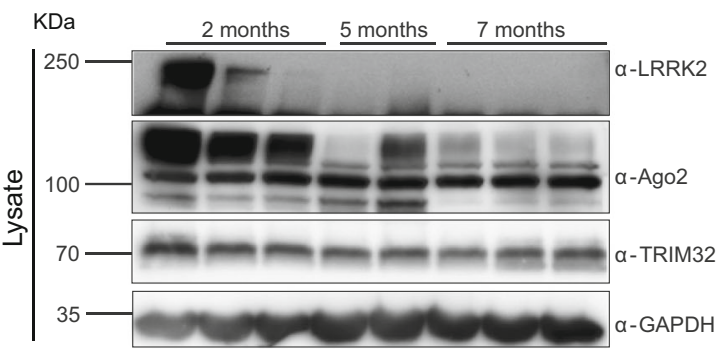

Fig. 1 TRIM32, LRRK2, and Ago2 form a complex. a HEK293T cells were transfected with plasmids for the overexpression of the indicated constructs. On the left panel, immunoblots of cell lysates, probed with the indicated antibodies, are shown. On the right panel, immunoprecipitations with anti-LRRK2 and anti-Ago2 antibodies are shown. The blots are

on these results, we conclude that LRRK2 and TRIM32 do not exclude each other at the Ago2-binding position, indicating that they most likely bind to different domains of the Ago2 protein.

\section{TRIM32 Antagonizes the Inhibition of miRNA Activity Induced by Pathogenic Mutants of LRRK2}

After showing that there is no binding antagonism between TRIM32 and LRRK2 (Fig. 2), we wanted to find out whether there might be a functional antagonism concerning
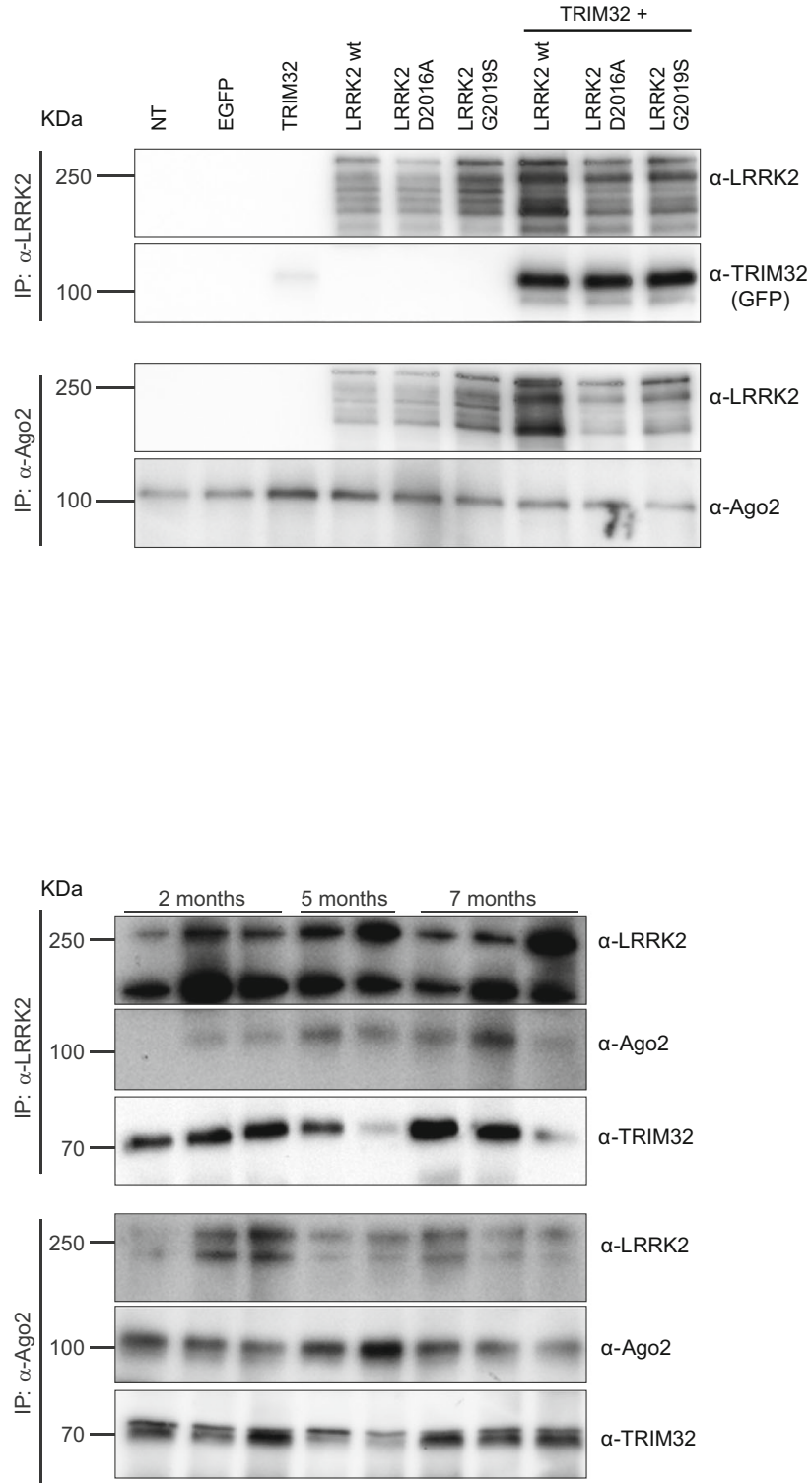

probed with the indicated antibodies. b On the left panel, immunoblots of lysates from brains of mice at different ages displaying the expression levels of endogenous LRRK2, Ago2, and TRIM32. On the right panel, immunoprecipitations with anti-LRRK2 and anti-Ago2 antibodies are shown. The blots are probed with the indicated antibodies

miRNA activity regulation. Therefore, we co-expressed sensors for Let-7a activity together with TRIM32 and wild-type LRRK2 or the two pathogenic LRRK2 mutant forms G2019S and R1441H in N2a cells. As previously reported, we were able to see increased miRNA activity, represented by a reduced luciferase reporter activity, in the presence of TRIM32 (Fig. 3). While expression of wild-type LRRK2 has no significant effect on the activity of Let-7a, expression of either LRRK2-G2019S or LRRK2-R1441H significantly reduced the activity of Let7a (represented by increased reporter activity, Fig. 3a, b). 
A
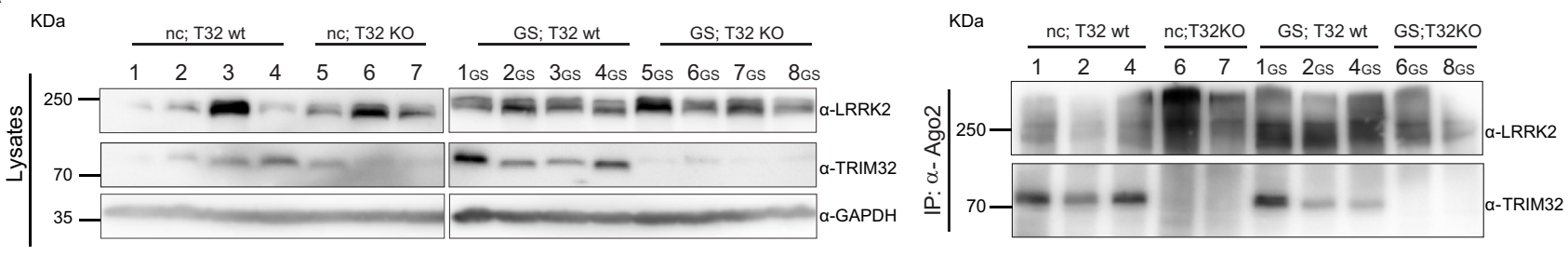

B

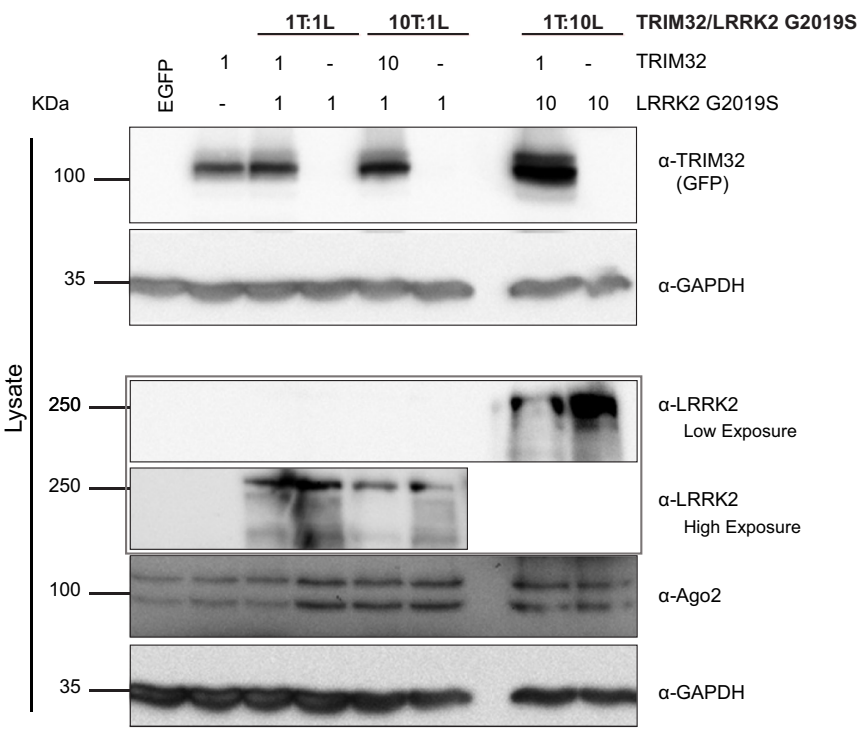

C

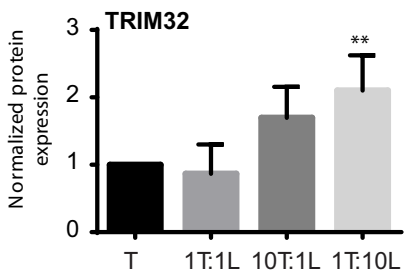

D

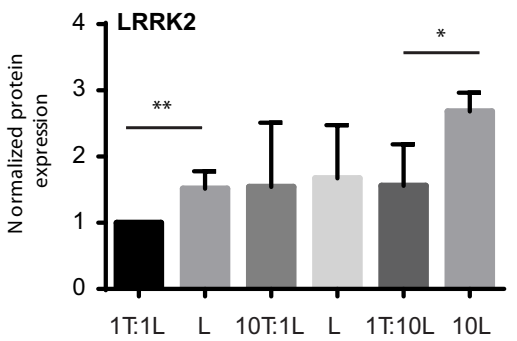

$E$

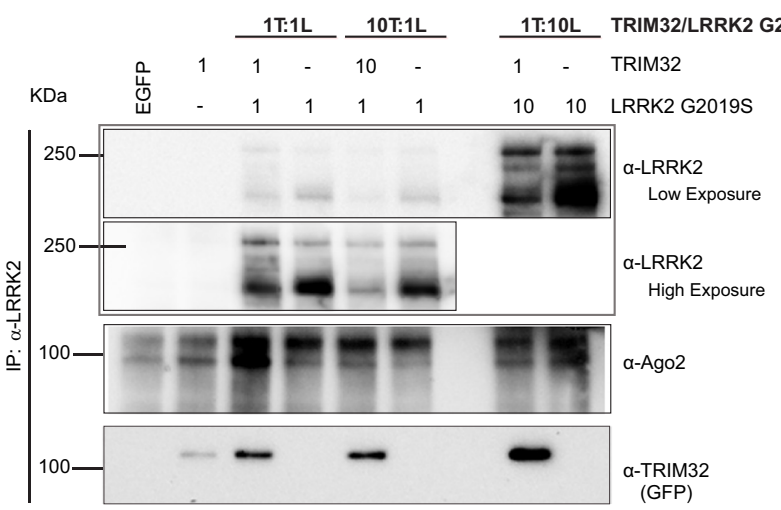

Fig. 2 TRIM32 and LRRK2 do not antagonize for binding at the RISC. a On the left panel, brain lysates obtained from adult mice with the indicated genotypes are shown. The blots are probed with the indicated antibodies. Each lane correspond to an individual mice identified with unique numbers. Abbreviations: $n c$ non-carrier, absence of mutations in the LRRK2 gene; GS LRRK2 G2019S mutation carriers; T32wt mice expressing TRIM32; T32KO mice lacking TRIM32 protein. On the right panel, immunoblots, probed with the indicated antibodies, after immunoprecipitation with an anti-Ago 2 antibody, are shown. b HEK293T cells were transfected with different ratios of plasmids for the overexpression of the indicated constructs. Abbreviations: $T$ GFPTRIM32, $L$ Flag-LRKK2 G2019S. Immunoblots from lysates of these cells showing the expression levels of LRRK2, TRIM32, and Ago2 are shown. In the case of LRRK2 detection, we show two different exposures

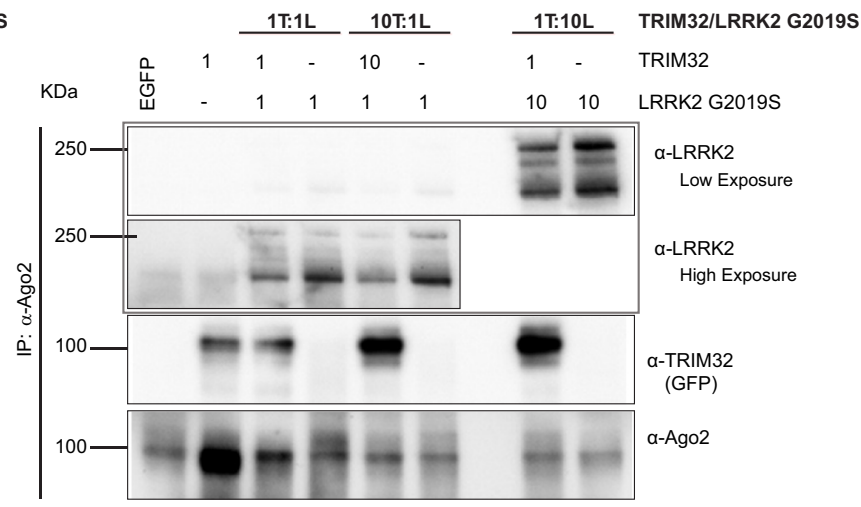

of the same blot, due to the high difference in the expression levels when LRRK2 has been transfected in a 1:1 vs. 1:10 times ratio. $\mathbf{c}$ Densitometric quantification of TRIM32 levels normalized to GAPDH expression levels; statistical differences were determined compared with the control conditions in which TRIM32 has been transfected without the presence of LRRK2. d Densitometric quantification of LRRK2 levels normalized to GAPDH expression levels; statistical analysis was performed compared with the control conditions in which LRRK2-G2019S has been transfected without the presence of TRIM32. e Immunoblots from immunoprecipitations with anti-LRRK2 or anti-Ago 2 antibodies from lysates of HEK293T cells, transfected as indicated in $\mathbf{b}$, are shown. The blots were probed with the indicated antibodies. Uncropped blots with high exposure, probed with the anti-LRRK2 antibody, are shown in the supplementary information (Supplementary Fig. 2A) 
A

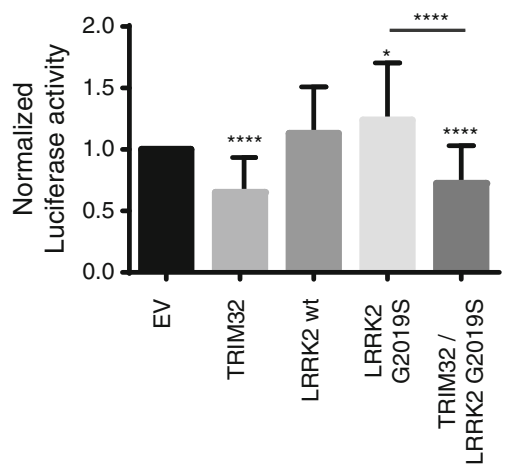

B

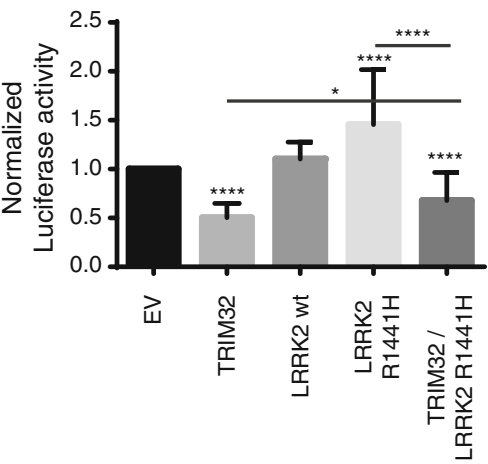

C

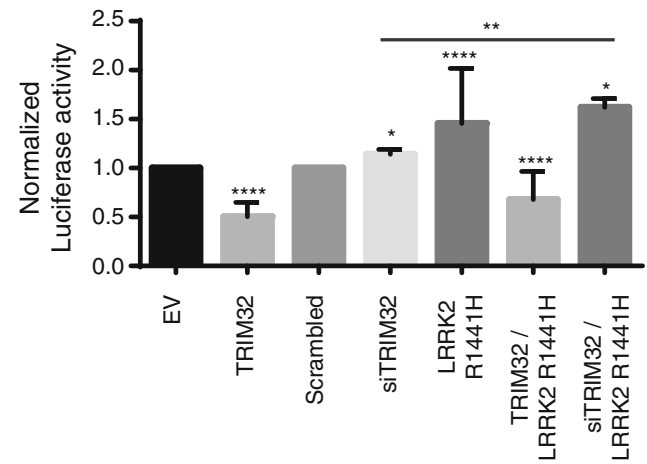

Fig. 3 TRIM32 antagonizes the pathogenic LRRK2 mutant-induced inhibition of Let-7a activity. a-c N2a cells were transfected with dualluciferase activity sensors for the activity of Let-7a. Additionally, the N2a

Strikingly, co-expression of TRIM32 together with LRRK2-G2019S or LRRK2-R1441H not only blocks their miRNA inhibition; it is even sufficient to induce activation of the miRNA Let-7a (Fig. 3a, b), although to a weaker extent than TRIM32 expression alone. Finally, we addressed the effect of a TRIM32 loss of function and observed that knockdown of TRIM32 inhibits the activity of Let-7a (Fig. 3c). Altogether, these data suggest that there is a functional antagonism between TRIM32 and pathogenic LRRK2 and that TRIM32 functions downstream of LRRK2.

\section{Pathogenic Mutant LRRK2 Inhibits TRIM32-Induced Neuronal Differentiation}

TRIM32 is well known for its function during neuronal cell fate specification [12, 13, 18, 25]. Since we have demonstrated here that pathogenic LRRK2 has an antagonistic function to TRIM32 during miRNA activity regulation, we speculated whether this also influences TRIM32induced neuronal differentiation. To address this question, we used a culture system of human neuroepithelial stem cells (NESCs) [26]. The NESCs were electroporated either with an empty vector only expressing GFP (EV, negative control), GFP-tagged TRIM32, or GFP-tagged TRIM32 together with Flag-tagged LRRK2-R1441H (Fig. 4). Electroporated cells were identified by GFP expression, and co-expression of LRRK2-R1441H was verified by staining with an anti-Flag antibody (Supplementary Fig. 3B). Neuronal differentiation was analyzed $48 \mathrm{~h}$ after electroporation by staining with the neuronal marker Tuj 1 . Additionally, we used Hoechst staining to analyze pyknotic nuclei, indicative of cell death. Previously, we have already shown that LRRK2 mutated at position 1441 inhibits cells were transfected with the indicated plasmids ( $E V$ empty vector). The diagrams show the detected normalized activity of the sensors. Statistical differences were determined with a Student $t$ test

neuronal differentiation [5]. As previously described, expression of TRIM32 was able to induce neuronal differentiation (Fig. 4a, b and supplementary Fig. 3A); in addition, expression of TRIM32 led to a marked increase in cell death (Fig. 4c and Supplementary Fig. 4). Both the induction of neuronal differentiation and the concomitant increase in cell death are significantly inhibited by co-expression of LRRK2-R1441H. Therefore, we conclude that pathogenic LRRK2 antagonizes not only the TRIM32-induced miRNA activity but also neuronal differentiation. Finally, since reduced neurite complexity is a well-known characteristic of in vitro cell culture models for PD [27], we also analyzed this feature. In the here used assay, expression of TRIM32 had no effect on neurite number, branching, or length. However, TRIM32 was also not able to antagonize the LRRK2-R1441H-induced reduction in neurite branching and length (Fig. 4d).

What do these results imply for the function of pathogenic LRRK2 during Parkinson's disease? We are convinced that our here presented results support the hypothesis that PD has a strong neurodevelopmental component. Based on our results, we conclude that pathogenic LRRK2 inhibits the activity of miRNAs in neural progenitor cells during brain development and therefore interferes with the precise timing of neuronal differentiation. At a first glance, it is counterintuitive that pathogenic LRRK2 also reduces the level of cell death that is induced by TRIM32-mediated neuronal differentiation. However, cell death, particularly correctly timed, is an important aspect of brain development. Disturbing the correct dynamics of neuronal differentiation and cell death might lead to an altered neuronal network more prone to neuronal degeneration at later stages. Additionally, we here identify TRIM32 as an interesting new modifier for PD, which might be relevant as a novel target for future therapeutic approaches. 

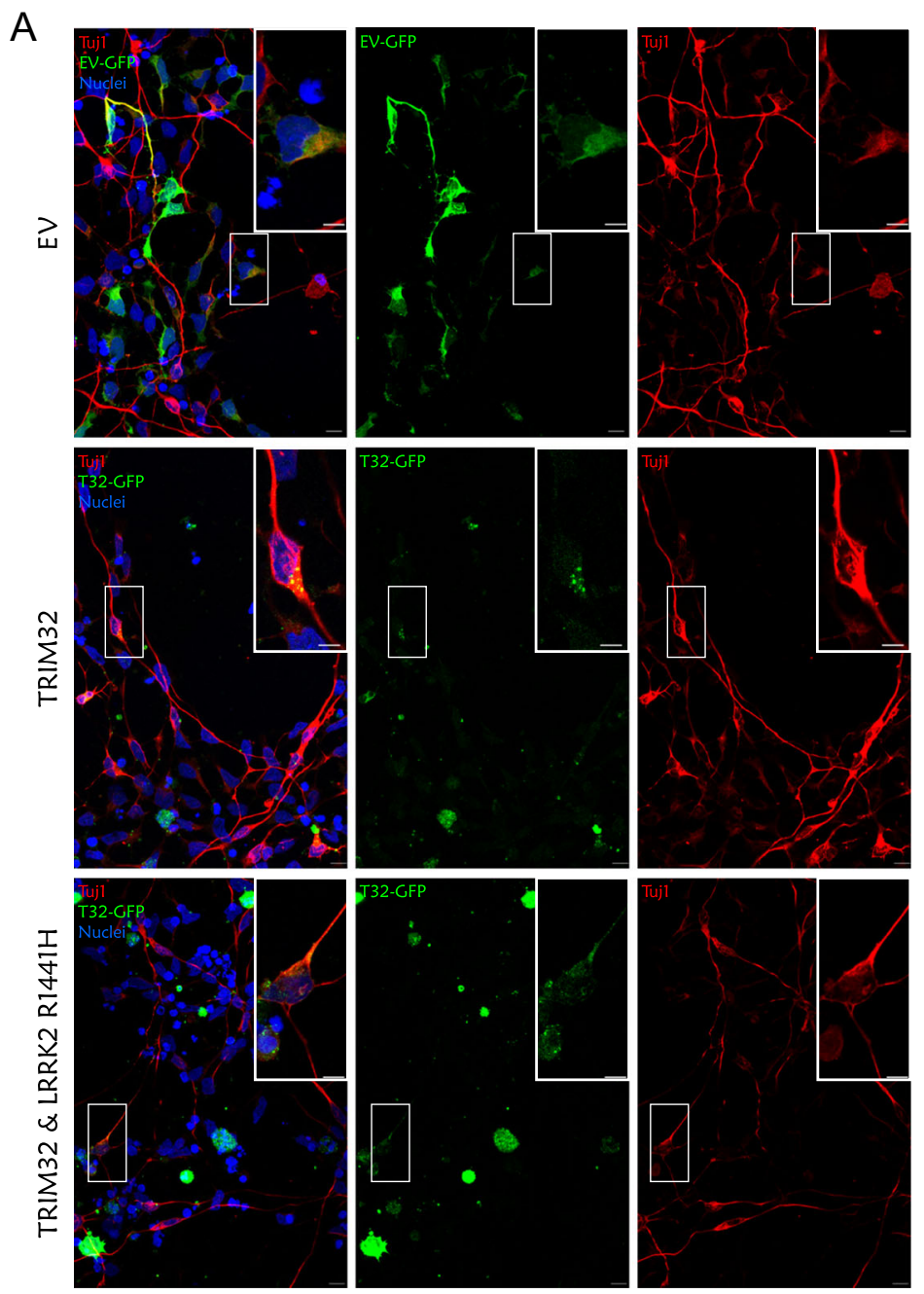

B
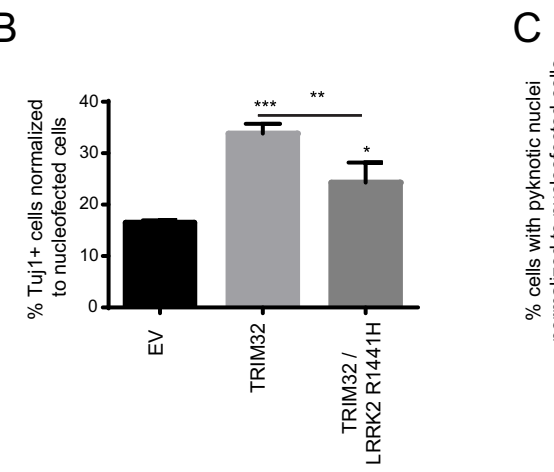

C
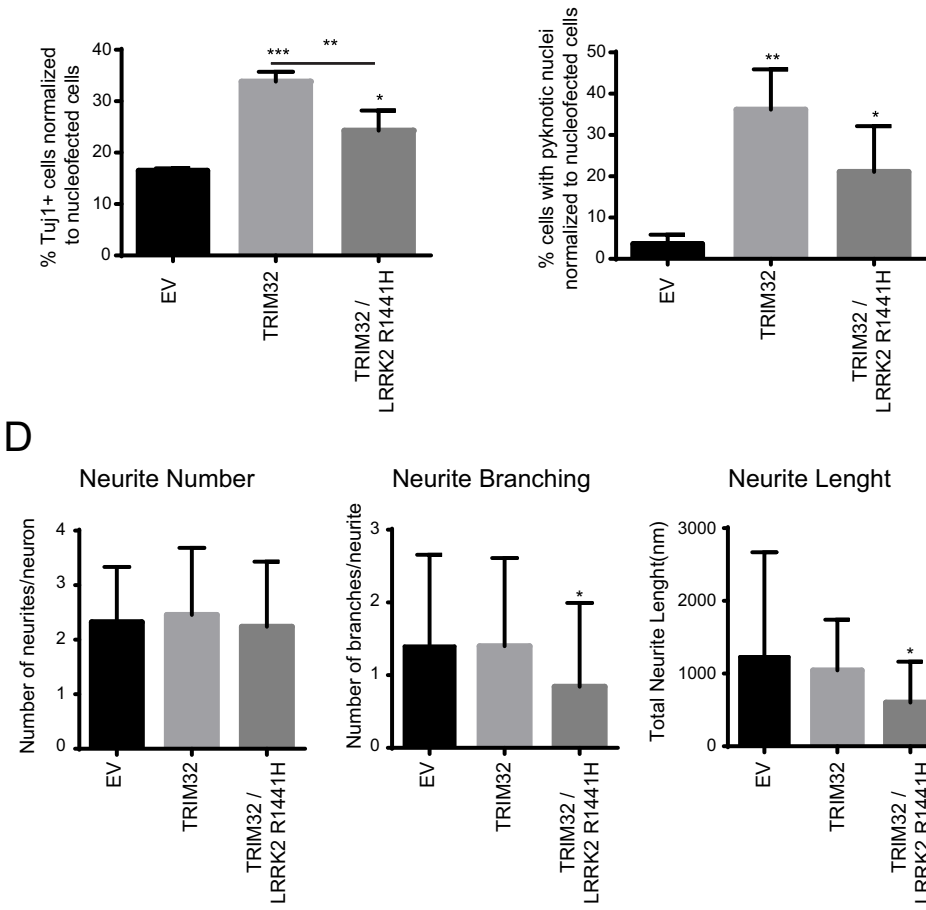

Neurite Lenght

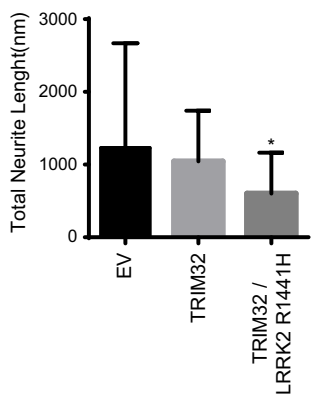


Fig. 4 Pathogenic LRRK2 inhibits TRIM32-induced neuronal differentiation. a-c Neuroepithelial stem cells were nucleofected with plasmids for the expression of GFP, TRIM32-GFP, or TRIM32-GFP + Flag-LRRK2-R1441H. After fixation, nucleofected cells (green) were stained with antibodies against $\mathrm{TuJ} 1$ (red) indicative of neuronal differentiation. DNA was visualized by staining with Hoechst. Neuronal differentiation (b), pyknotic nuclei indicating cell death (c), and neurite complexity (neurite number, branching, and length) in transfected cells were quantified. Statistical differences were determined using an unpaired $t$ test $(* P<0.05, * * P<0.01, * * * P<0.005)$

\section{Materials and Methods}

\section{Cell Lines, Culture Conditions, and Transfections}

Neuroblastoma (N2a) and human embryonic kidney (HEK) 293T cells were maintained in DMEM (Sigma) supplemented with $10 \%$ heat-inactivated fetal bovine serum (FBS), $200 \mathrm{mM}$ L-glutamine, and penicillin/streptomycin in a $5 \% \mathrm{CO}_{2}$-humidified atmosphere at $37^{\circ} \mathrm{C}$.

For protein analysis, cells were seeded onto non-coated plates at high density the day before transfection. The following day, cells were transfected with the indicated plasmids using TurboFect (Fermentas) according to the manufacturer's recommendations.

In order to assess neuronal differentiation potential, we used human NESCs [26]. Cells were seeded onto matrigelcoated coverslips under maintenance culture conditions, and 18-24 h later, they were transfected in adherence conditions with a 4D-NucleofectorTM Y Unit (LONZA). Following the manufacturer's instructions, cells were transfected with a total amount of $24 \mu \mathrm{g}$ of DNA diluted into AD1 4D-Nucleofector ${ }^{\mathrm{TM}} \mathrm{Y}$ solution using program ER-137. Combinations of pmaxGFP vector as control, pBABE-Flag, pcDNA3-GFP-Myc(N-Term)-TRIM32, and pCMV-Flag-LRRK2-R1441H were used for transfections. In order to induce neuronal differentiation, $24 \mathrm{~h}$ after transfection, culture media were completely exchanged with differentiation media containing $1 \mu \mathrm{l}$ PMA, $10 \mu \mathrm{l}$ hBDN, $10 \mu \mathrm{l}$ hGDNF, $100 \mu \mathrm{l}$ ascorbic acid, $1 \mu \mathrm{l}$ TGFb3, and $50 \mu \mathrm{dbcAMP}(10 \mathrm{ml})$. Cells were incubated for $60-72 \mathrm{~h}$ and then fixed in 4\% PFA for $15 \mathrm{~min}$ at RT.

\section{Mice and Tissue Samples}

Mouse husbandry was conducted in accordance with the existing Luxembourgish regulations for the protection of animals used for scientific purposes. TRIM32 $+/-$ mice in a C57BL/6N background [23] were backcrossed with either LRRK2-G2019S or LRRK2 R1441G hemizygous mice (JAX® Mice) for at least five generations to generate the double-mutant animals with an enriched FvB background. Once a more pure FvB background was reached for all the genotypes used in this study we proceeded with the breedings to obtain the control and experimental animals. For that purpose, TRIM32 +/- mice, were crossed with TRIM32+/-; hemizygous LRRK2-G2019S or LRRK2R1441G mice, obtaining hemizygous LRRK2-G2019S; TRIM32 -/- and hemizygous LRRK2-R1441G: TRIM32 -/mice, respectively.

Genotyping of the animals was performed as previously described [23] or accordingly with the protocol defined by the provider (JAX).

Mouse brain samples were obtained from animals with the indicated genotypes and ages. For tissue collection, mice were euthanized according to the Luxembourgish Law for animal experimentation, and brains were dissected and deep frozen in liquid nitrogen and stored at $-80^{\circ} \mathrm{C}$ for subsequent protein extraction. For immunoblotting analysis, brain samples were homogenized using syringes of decreasing sizes in lysis buffer on ice until obtaining a cellular suspension that was subsequently treated as a cellular lysate.

\section{Western Blot and Immunoprecipitation}

For Western blot and immunoprecipitation assays, protein extraction from brain lysates and cells was performed with lysis buffer consisting of $2 \%$ Triton X-100 and complete protease inhibitor cocktail (PIC) (Roche) in phosphate-buffered saline (PBS) supplemented with $3 \mu \mathrm{l} / \mathrm{ml}$ RNAse A. In order to detect protein-protein interactions, equal amounts of protein were incubated for $4 \mathrm{~h}$ with the indicated precipitating antibodies at $4{ }^{\circ} \mathrm{C}$, followed by overnight incubation with protein-G agarose beads (GE Healthcare). Subsequently, protein complexes were eluted by boiling the samples for $5 \mathrm{~min}$ at $99{ }^{\circ} \mathrm{C}$ in protein sample buffer.

Protein lysates and protein complexes from the immunoprecipitation assays were resolved by SDS-PAGE, and immunoblotting was performed. Nitrocellulose membranes were incubated at $4{ }^{\circ} \mathrm{C}$ with the following primary antibodies: rabbit anti-LRRK2 (MJFF2(c41-2)) (Abcam, ab133474), rabbit anti-TRIM32 (\#3149 and \#3150, [17]), rabbit anti-GAPDH (D16H11) XP (Cell Signaling, \#5174), and rabbit anti-Ago2/eIF2C2 (Abcam, ab32381). Membranes were incubated with the appropriate HRPcoupled secondary antibodies (GE Healthcare), and the enhanced chemiluminescence signal was detected with SuperSignal West Pico Chemiluminescent Substrate (Pierce, Thermo Scientific). Protein content was visualized with a STELLA 3200 Bio/Chemiluminescence modular imaging system (RAytest) device, acquiring several exposure times for each membrane.

Quantification was completed using ImageJ and its FIJI plugin; all data shown are normalized to the intensity of GAPDH, which had been used as loading control. 


\section{Luciferase Assays}

N2a cells were seeded at a density of $10^{5}$ cells per well in 24well plates. The following day, cells were transfected using TurboFect (Fermentas) according to the manufacturer's instructions. To determine the activity of miRNA Let-7a, each experimental condition contained $0.25 \mu \mathrm{g}$ of pcDNA3.1DLet-7a-1 [12] together with $0.2 \mu \mathrm{g}$ of pmirGLO-let7 [28]. Up to $0.6 \mu \mathrm{g}$ was completed with a 1:1 ratio of each of the indicated plasmids: pBABE-Flag, pcDNA3-GFP-Myc $(\mathrm{N}-$ Term)-TRIM32, pCMV-Flag-LRRK2-wt, pCMV-FlagLRRK2-G2019S, pCMV-Flag-LRRK2-R1441H, pRNATH1.4/Retro-GFP(IRES)-scrambled sequence (siRNA12), pRNAT-H1.4/Retro-GFP(IRES)-TRIM32 (siRNA 2), and pRNAT-H1.4/Retro-GFP(IRES)-TRIM32 (siRNA 5) (GenScript). Forty-eight after transfection, cells were lysed and firefly and renilla luciferase activities were measured using the Dual-Luciferase Assay System (Promega) according to the manufacturer's instructions in a Microplate Reader Infinite M200Pro-LCSB0414 (TECAN). The ratio of firefly luciferase (FL) activity to renilla luciferase (RL) was determined for each reaction, and all values were normalized to those of the empty vector.

\section{Immunostaining and Confocal Analysis}

Fixed cells were permeabilized with $0.5 \%$ Triton X-100 in PBS, 15 min at RT, blocked with $10 \%$ FBS for $1 \mathrm{~h}$ at RT, and incubated o/n at $4{ }^{\circ} \mathrm{C}$ with antibodies against LRRK2 (Rabbit, Abcam, ab133474 (MJFF2(c41-2)), neuron-specific class III B-tubulin (Tuj1) (mouse, Covance, PRB-435P). Immunofluorescent detection was carried out with the corresponding Alexa Fluor@ 568 secondary antibodies, and nuclei were counterstained with Hoechst 33342 Solution (Invitrogen).

Tile scan pictures of random areas across the coverslips were acquired with a $\times 63$ magnification in a confocal microscope LSM 710/Observer Z1-LCSB0451 (Zeiss). Quantification of the number of nuclei was done using ImageJ ITCN Plugin; however, the number of neurons and pyknotic nuclei was determined manually counting a minimum of 2000 cells for each experimental replicate and condition from three independent experiments. To determine statistical differences, unpaired two-tailed $t$ test was performed.

\section{Statistical Analysis}

In order to determine statistical differences in the different experiments, unpaired Student's $t$ test was performed. At least three independent experiments were analyzed in each case and $P$ values smaller than 0.05 were considered significant.
Acknowledgements The authors would like to thank Myriam Gorospe (National Institute on Aging, NIH), Sven Diederichs (Deutsches Krebsforschungszentrum), Germana Meroni (Telethon Institute of Genetics and Medicine), and Frank Gillardon (Boehringer Ingelheim $\mathrm{GmbH}$ ) for plasmids, and Thea van Wuellen and Inga Werthschulte for excellent technical assistance. We further acknowledge the support through the Pluripotent Stem Cell Facility at the LCSB.

Authors' Contributions LGC contributed to the design and performance of experiments, collection, and assembly of data, data analysis, interpretation, and manuscript writing. IM, JT, and SN performed experiments, collected and analyzed data, and drafted parts of the manuscript. JCS planned experiments, analyzed data, provided advice, and revised the manuscript. All authors have been involved in drafting the manuscript or revising it critically for important intellectual content. All authors approved the final version of the manuscript and agreed to be accountable for all aspects of the work.

\section{Compliance with Ethical Standards}

Funding This project was supported by the LCSB pluripotent stem cell core facility. The JCS lab is supported by the Fonds National de la Recherche (FNR) Luxembourg (CORE, C13/BM/5791363) and The Dementia Consortium: Alzheimer's UK, Eisai, Lilly, and MRCT. This is an EU Joint Programme-Neurodegenerative Disease Research (JPND) project (INTER/JPND/14/02; INTER/JPND/15/11092422). Further support comes from the SysMedPD project which has received funding from the European Union's Horizon 2020 research and innovation program under grant agreement No. 668738. Finally, we also thank the private donors who support our work at the Luxembourg Centre for Systems Biomedicine.

Open Access This article is distributed under the terms of the Creative Commons Attribution 4.0 International License (http:// creativecommons.org/licenses/by/4.0/), which permits unrestricted use, distribution, and reproduction in any medium, provided you give appropriate credit to the original author(s) and the source, provide a link to the Creative Commons license, and indicate if changes were made.

\section{References}

1. Beitz JM (2014) Parkinson's disease: a review. Front Biosci (Scholar edition) 6:65-74

2. Moustafa AA, Chakravarthy S, Phillips JR, Gupta A, Keri S, Polner B, Frank MJ, Jahanshahi M (2016) Motor symptoms in Parkinson's disease: a unified framework. Neurosci Biobehav Rev 68:727-740

3. Stirpe P, Hoffman M, Badiali D, Colosimo C (2016) Constipation: an emerging risk factor for Parkinson's disease? Eur J Neurol 23: $1606-1613$

4. Sveinbjornsdottir S (2016) The clinical symptoms of Parkinson's disease. J Neurochem 139(Suppl 1):318-324

5. Bahnassawy L, Nicklas S, Palm T, Menzl I, Birzele F, Gillardon F, Schwamborn JC (2013) The Parkinson's disease associated LRRK2 mutation R1441G inhibits neuronal differentiation of neural stem cells. Stem Cells Dev 22(18):2487-2496

6. Garcia-Reitboeck P, Anichtchik O, Dalley JW, Ninkina N, Tofaris GK, Buchman VL, Spillantini MG (2013) Endogenous alphasynuclein influences the number of dopaminergic neurons in mouse substantia nigra. Exp Neurol 248:541-545

7. Le Grand JN, Gonzalez-Cano L, Pavlou MA, Schwamborn JC (2015) Neural stem cells in Parkinson's disease: a role for neurogenesis defects in onset and progression. Cell Mol Life Sci CMLS 72:773-797 
8. Verstraeten A, Theuns J, Van Broeckhoven C (2015) Progress in unraveling the genetic etiology of Parkinson disease in a genomic era. Trends Genet TIG 31:140-149

9. Cookson MR (2010) The role of leucine-rich repeat kinase 2 (LRRK2) in Parkinson's disease. Nat Rev Neurosci 11:791-797

10. Healy DG, Falchi M, O'Sullivan SS, Bonifati V, Durr A, Bressman S, Brice A, Aasly J et al (2008) Phenotype, genotype, and worldwide genetic penetrance of LRRK2-associated Parkinson's disease: a case-control study. Lancet Neurol 7:583-590

11. Gehrke S, Imai Y, Sokol N, Lu B (2010) Pathogenic LRRK2 negatively regulates microRNA-mediated translational repression. Nature 466:637-641

12. Nicklas S, Okawa S, Hillje AL, Gonzalez-Cano L, Del Sol A, Schwamborn JC (2015) The RNA helicase DDX6 regulates cellfate specification in neural stem cells via miRNAs. Nucleic Acids Res 43:2638-2654

13. Schwamborn JC, Berezikov E, Knoblich JA (2009) The TRIMNHL protein TRIM32 activates microRNAs and prevents selfrenewal in mouse neural progenitors. Cell 136:913-925

14. Reymond A, Meroni G, Fantozzi A, Merla G, Cairo S, Luzi L, Riganelli D, Zanaria E et al (2001) The tripartite motif family identifies cell compartments. EMBO J 20:2140-2151

15. Napolitano LM, Meroni G (2012) TRIM family: pleiotropy and diversification through homomultimer and heteromultimer formation. IUBMB Life 64:64-71

16. Gonzalez-Cano L, Hillje AL, Fuertes-Alvarez S, Marques MM, Blanch A, Ian RW, Irwin MS, Schwamborn JC et al (2013) Regulatory feedback loop between TP73 and TRIM32. Cell Death Dis 4:e704

17. Hillje AL, Worlitzer MM, Palm T, Schwamborn JC (2011) Neural stem cells maintain their Stemness through protein kinase C zetamediated inhibition of TRIM32. Stem Cells (Dayton, Ohio) 29: 1437-1447

18. Hillje AL, Pavlou MA, Beckmann E, Worlitzer MM, Bahnassawy L, Lewejohann L, Palm T, Schwamborn JC (2013) TRIM32dependent transcription in adult neural progenitor cells regulates neuronal differentiation. Cell Death Dis 4:e976
19. Pavlou MA, Colombo N, Fuertes-Alvarez S, Nicklas S, Cano LG, Marin MC, Goncalves J, Schwamborn JC (2016) Expression of the Parkinson's disease-associated gene alpha-synuclein is regulated by the neuronal cell fate determinant TRIM32. Mol Neurobiol

20. Sarraf SA, Raman M, Guarani-Pereira V, Sowa ME, Huttlin EL, Gygi SP, Harper JW (2013) Landscape of the PARKIN-dependent ubiquitylome in response to mitochondrial depolarization. Nature 496:372-376

21. West AB, Moore DJ, Biskup S, Bugayenko A, Smith WW, Ross CA, Dawson VL, Dawson TM (2005) Parkinson's diseaseassociated mutations in leucine-rich repeat kinase 2 augment kinase activity. Proc Natl Acad Sci U S A 102:16842-16847

22. Maekawa T, Kubo M, Yokoyama I, Ohta E, Obata F (2010) Agedependent and cell-population-restricted LRRK2 expression in normal mouse spleen. Biochem Biophys Res Commun 392:431-435

23. Nicklas S, Otto A, Wu X, Miller P, Stelzer S, Wen Y, Kuang S, Wrogemann K et al (2012) TRIM32 regulates skeletal muscle stem cell differentiation and is necessary for normal adult muscle regeneration. PLoS One 7:e30445

24. Li X, Patel JC, Wang J, Avshalumov MV, Nicholson C, Buxbaum JD, Elder GA, Rice ME et al (2010) Enhanced striatal dopamine transmission and motor performance with LRRK2 overexpression in mice is eliminated by familial Parkinson's disease mutation G2019S. J Neurosci 30:1788-1797

25. Sato T, Okumura F, Kano S, Kondo T, Ariga T, Hatakeyama S (2011) TRIM32 promotes neural differentiation through retinoic acid receptor-mediated transcription. J Cell Sci 124:3492-3502

26. Reinhardt P, Glatza M, Hemmer K, Tsytsyura Y, Thiel CS, Hoing S, Moritz S, Parga JA et al (2013) Derivation and expansion using only small molecules of human neural progenitors for neurodegenerative disease modeling. PLoS One 8:e59252

27. Thomas JM, Li T, Yang W, Xue F, Fishman PS, Smith WW (2017) 68 and FX2149 attenuate mutant LRRK2-R1441C-induced neural transport impairment. Front Aging Neurosci 8:337

28. Guo R, Abdelmohsen K, Morin PJ, Gorospe M (2013) Novel MicroRNA reporter uncovers repression of let-7 by GSK-3beta. PLoS One 8:e66330 